\title{
Laparoscopic aspirator bracket: A new instrument facilitating the aspiration and exposure of operative field simultaneously in laparoscopic nephron- sparing surgery
}

\section{Fengqi Yan}

Tangdu Hospital Fourth Military Medical University: Air Force Medical University Tangdu Hospital

\section{Xiaoliang Dou}

Tangdu Hospital Fourth Military Medical University: Air Force Medical University Tangdu Hospital

\section{Guangfeng Zhu}

Tangdu Hospital Fourth Military Medical University: Air Force Medical University Tangdu Hospital

\section{Qisheng Tang}

Tangdu Hospital Fourth Military Medical University: Air Force Medical University Tangdu Hospital

\section{Bo Zhang}

Tangdu Hospital Fourth Military Medical University: Air Force Medical University Tangdu Hospital

\section{Bo Zhao}

Bao Ji People's Hospital

Lei Yu

Fourth Military Medical University: Air Force Medical University

\section{He Wang}

Tangdu Hospital Fourth Military Medical University: Air Force Medical University Tangdu Hospital

\section{Yong Wang ( $\nabla$ dryongwangfmmu@yeah.net)}

Tangdu Hospital Fourth Military Medical University: Air Force Medical University Tangdu Hospital https://orcid.org/0000-0003-0477-2019

\section{Research Article}

Keywords:

Posted Date: March 2nd, 2022

DOl: https://doi.org/10.21203/rs.3.rs-917427/v1

License: (9) This work is licensed under a Creative Commons Attribution 4.0 International License. Read Full License 


\section{Abstract \\ Background}

Laparoscopic nephron-sparing surgery (NSS) is the standard of care for small renal masses whenever feasible. This study aims to describe a novel laparoscopic aspirator bracket (LAB) and its use in laparoscopic NSS by a simple enucleation technique.

\section{Methods}

Between July 2017 and July 2019, we performed 54 laparoscopic NSS due to renal tumour using an SE technique by either LAB $(n=26)$ or laparoscopic aspirator $(\mathrm{LA}, n=28)$ in 3 independent medical centres by 6 experienced surgeons. The novel aspirator could aspirate liquid and expose the operative field simultaneously. The details of operative technique are provided in the accompanying video. General characteristics and perioperative data of all patients were collected and retrospectively. Patients were followed up for 12 months after laparoscopic renal tumor simple enucleation (SE) operation. Renal function outcomes and perioperative complications were assessed.

\section{Results}

All 54 patients were successfully operated without conversion to open surgery. The use of the LAB in laparoscopic renal tumor SE operation can shorten the warm ischemia time ([WIT], LAB 12.92 \pm 8.38 min vs. LA $17.89 \pm 6.73 \mathrm{~min}$ ) and increase the zero ischemia (ZI) rate (LAB $23.08 \%$ vs. LA $3.57 \%)$. In the oneyear follow-up, the LAB group showed quicker renal function recovery (ipsilateral renal function 3 months after the surgery, LAB $41.67 \pm 10.11 \%$ vs. LA $36.75 \pm 10.30 \%)$. The limitations of this study were limited number of patients and retrospective attribute.

\section{Conclusion}

The new LAB could aspirate and expose the operative field with a single instrument. In operations that need to expose and aspirate simultaneously, such as in renal tumor simple enucleation, it could shorten WIT and improve the postoperative renal function recovery.

\section{Introduction}

Over the past four decades, laparoscopic and robot-assisted laparoscopic operation have become the mainstay of urologic operations [1]. However, the exposure of operative field during surgery is a constant problem, especially in surgical fields full of liquids, such as blood, urine, lymph, or melted fat, and the surgeon has to manipulate a pair of laparoscopic forceps in one hand for exposure and a laparoscopic ultrasound knife or a pair of bipolar forceps in the other hand for hemostasis. To aspirate the liquids in 
the operative field, the surgeon needs to exchange the laparoscopic forceps for a laparoscopic aspirator or add an assistant trocar, which will increase the operative time, bleeding volume, or operative trauma. For some special operations, such as renal mass enucleation, the elongated, warm ischemia time (WIT) may result in worse renal function [2], or the surgeon could add another trocar to allow the assistant surgeon to manipulate the aspirator [3]. In such cases, the operative space may be congested by operative instruments, thus causing instrument collision. In addition, the assistant trocar adds to the surgical incision.

How can the surgeon expose the operative field and aspirate the liquid simultaneously without congesting the operative field? Our idea was to combine the function of forceps and aspirator in one instrument. A new surgical instrument-a laparoscopic aspirator bracket (LAB) made of silicone rubberwas developed. It was put at the end of the laparoscopic aspirator, thus making it applicable for surgical exposure and aspiration simultaneously, especially suitable for very narrow region and operative filed with full of liquids.

In this study, the new aspirator bracket was applied in a series of operations in combination with the laparoscopic aspirator, such as for simple laparoscopic renal tumor enucleation. Perioperative data were compared with traditional laparoscopic renal tumor enucleation.

\section{Materials And Methods}

\section{Clinical data}

This study was approved by the Ethics Committee of Tang Du Hospital (April, 2017). Written consent was signed by all the participants included in the study. All patients were evaluated on a protocol that had been approved by the institutional review board.

From July 2017 to July 2019 , a total of $54 \mathrm{CT}_{1}-\mathrm{CT}_{2}$ renal cell carcinoma patients were operated by laparoscopic surgery in 3 hospitals (Tang Du hospital, Xi Jing hospital and Bao Ji People's Hospital) by 6 experienced urologists (Mr. Yong Wang, Mr. Bo Zhang, Mr. Jian Jun Ma, Mr. Lei Yu, Mr. He Wang, and Mr. Bo Zhao). Each surgeon had more than 100 cases of experience in laparoscopic renal mass enucleation. Their peri-operative clinical data were documented and retrospectively analysed. All the patients were diagnosed with renal tumor mass by enhanced computed tomography (CT) scan before surgery. Twentysix of them were operated with the LAB. Twenty-eight patients were operated with traditional laparoscopic aspirator (LA) and forceps.

The following demographic characteristics of the patients were collected: age, sex, body mass index (BMI), location of tumor, size of tumor, Radius Exophytic Nearness Anterior/Posterior Location (RENAL) score and tumour histology. In addition, the operation time, WIT, estimated blood loss volume, intraoperative transfusion, complications, and pseudo capsule damage were recorded and analysed. The operation time was defined as the period from skin incision to closure, the WIT is the time from clamping 
the renal artery to releasing the renal artery clamp. All the patients were followed up for at least 1 year after surgery, and the serum creatine, eGFR, and ipsilateral renal function values of patients were measured and analysed before surgery and at the 3rd, 6th, 9th and 12th months after surgery respectively.

\section{Surgical Instruments}

The LAB is an invention (ZL 201620736850.2 from State Intellectual Property Office of P.R. China) developed by Mr. Yong Wang (Fig. 1, A and B). It is made of S820 silica gel (Xi Rui Bo Technology Co ITD, Wu Han, P.R. China) by Chang Ping Industrial Incorporation (Xing Ping County, Shaan'xi Province, P.R. China). It needs a 12-mm standard laparoscopic trocar to pass through. In both groups, all the laparoscopic instruments were the same. The 5-mm laparoscopic aspirator (№. 101.149) was made by Kang Ji Medical Instruments Ltd. (Hang Zhou, Zhe Jiang Province, P.R. China)

\section{Surgical Procedure}

The tumor was enucleated with a simple enucleation technique [2]. That is, it was incised to open the parenchyma along the tumor margin, bluntly separated the tumor and renal parenchyma along the pseudocapsule by closed laparoscopic scissors. To expose the cleavage plane, the tumor was pushed aside with either the LAB (LAB group) or the forceps (LA group). The large vessels traversing this surgical plane were cut with laparoscopic scissors and then clipped in the surgical plane when needed. The whole tumor was enucleated from the kidney along the cleavage plane. The bleeding site was ablated with bipolar forceps. Running suture was used for the tumor bed, with $2-0$ V-Loc ${ }^{\text {TM }}$ sutures (Medtronic, Inc., Shang Hai, P.R. China). For high Radius Exophytic Nearness Anterior/Posterior Location (RENAL) score tumors [4], a laparoscopic ultrasound was used to locate the tumor and determine the distance between the tumor and renal vessels or the collection system. The peri-operative data were documented.

For the LA group, the surgeon (right-handed) had laparoscopic forceps in the left hand and the laparoscopic scissors in the right hand for cutting. When too much blood was seen in the surgical cleavage plane between tumor and parenchyma, the forceps were withdrawn and the aspirator was inserted (Fig. 2, A). If the surgical cleavage plane was clear, the aspirator was withdrawn and the scissors were inserted. The whole tumor was enucleated by a combination of cutting and blunt separation by the scissors [2].

For the LAB group, before enucleation of the renal tumor, the bracket was put on the end of the laparoscopic aspirator. The surgeon had the LAB in one hand for exposure and aspiration and the laparoscopic scissors in the other hand for cutting (Fig. 2, B). The renal capsule was cut open with the scissors (Fig. 2, C) and the tumor was pushed aside with the LAB. The cleavage plane was found by a combination of cutting and blunt separation with the scissors (Fig. 2, D), and the blood was aspirated 
with the aspirator whenever needed. The whole tumor was then enucleated along the cleavage plane (Video 1).

\section{Statistical analysis}

Descriptive analyses of patient characteristics, perioperative data, and postoperative follow-up data were conducted, including central tendency and dispersion (mean \pm standard deviation [SD]), or median and frequency distribution. The outcomes were analysed using a t-test or $\chi^{2}$ test, and a $p$-value $<0.05$ was considered statistically significant. SPSS version 18.0 (SPSS, Inc., Chicago, IL) was used for statistical analysis.

\section{Results}

The demographic characteristics of the patients are shown in Table 1. A total of 54 patients were enrolled in this study. The mean age of the patients $59.52 \pm 11.28$ years old, with a median age of 59 years. Among all patients, 40 were males, and 14 were females. The mean BMI was $24.56 \pm 4.75 \mathrm{~kg} / \mathrm{m}^{2}$. Of all the patients, 29 patients had the tumor on the right side and 25 on the left side. and the tumor average diameter $_{\max }$ is $4.03 \pm 1.60 \mathrm{~cm}$. The RENAL score was $6.48 \pm 1.91$ points. The pathological results showed that among 54 patients, ccRCC accounted for 45, Papillary type accounted for 5, Chromophobe accounted for 3 , and Oncocytoma accounted for 1 . Fifty-four patients were randomly assigned to the $L A B$ group and the LA group, of which 26 were in the LAB group and 28 were in the LA group. And, there were no difference in demographic characteristics between the two groups of patients (Table 1). 
Table 1

Baseline characteristics for patients involved in this research

\begin{tabular}{|c|c|c|c|c|}
\hline Item & $\begin{array}{l}\text { All } \\
(N=54)\end{array}$ & $\begin{array}{l}\text { LAB group } \\
(n=26)\end{array}$ & $\begin{array}{l}\text { LA group } \\
(n=28)\end{array}$ & $p$ value \\
\hline \multicolumn{5}{|l|}{ Age (years) } \\
\hline Mean \pm SD & $59.52 \pm 11.28$ & $59.61 \pm 11.84$ & $59.43 \pm 10.95$ & 0.952 \\
\hline Median & 59.0 & 62.5 & 57.5 & \\
\hline \multicolumn{5}{|l|}{ Sex } \\
\hline Male & 40 & 20 & 20 & \multirow[t]{2}{*}{0.645} \\
\hline Female & 14 & 6 & 8 & \\
\hline $\operatorname{BMI}\left(\mathrm{Kg} / \mathrm{m}^{2}\right)$ & $24.56 \pm 4.75$ & $23.92 \pm 5.29$ & $25.16 \pm 4.19$ & 0.340 \\
\hline \multicolumn{5}{|l|}{ Side } \\
\hline Right & 29 & 15 & 14 & \multirow[t]{2}{*}{0.571} \\
\hline Left & 25 & 11 & 14 & \\
\hline Diameter $_{\max }(\mathrm{cm})$ & $4.03 \pm 1.60$ & $4.00 \pm 1.85$ & $4.05 \pm 1.38$ & 0.923 \\
\hline RENAL score & $6.48 \pm 1.91$ & $6.88 \pm 2.01$ & $6.11 \pm 1.77$ & 0.137 \\
\hline $\mathrm{R}$ & $1.56 \pm 0.66$ & $1.58 \pm 0.70$ & $1.54 \pm 0.64$ & 0.822 \\
\hline$E$ & $1.43 \pm 0.66$ & $1.50 \pm 0.65$ & $1.36 \pm 0.68$ & 0.433 \\
\hline$N$ & $1.59 \pm 0.79$ & $1.61 \pm 0.80$ & $1.58 \pm 0.79$ & 0.840 \\
\hline \multirow[t]{2}{*}{ A } & $A:(18 / 54)$ & \multirow[t]{2}{*}{$A:(8 / 26) P:(13 / 26)$} & \multirow[t]{2}{*}{$A:(10 / 28) P:(9 / 28)$} & \multirow[t]{2}{*}{0.356} \\
\hline & $P:(22 / 54)$ & & & \\
\hline $\mathrm{L}$ & $1.59 \pm 0.57$ & $1.54 \pm 0.58$ & $1.64 \pm 0.56$ & 0.504 \\
\hline \multicolumn{5}{|l|}{ Tumour histology } \\
\hline ccRCC & 45 & 22 & 23 & \\
\hline Papillary type & 5 & 2 & 3 & \multirow[t]{2}{*}{0.686} \\
\hline Chromophobe & 3 & 1 & 2 & \\
\hline Oncocytoma & 1 & 1 & 0 & \\
\hline
\end{tabular}

All the procedures were performed successfully in 3 hospitals by 6 experienced urologists. The operative data of the patients from two groups were compared. As can be seen in Table 2, there were no differences between the two groups regarding total operative time, median blood loss, intra-operative transfusion rate 
and perioperative complications. However, the warm ischemia time (WIT) of the LA group was $17.89 \pm$ $6.73 \mathrm{~min}$, while the WIT of the LAB group was only $12.92 \pm 8.38 \mathrm{~min}$, which was much shorter than that of the LA group. In addition, only 1 of the 28 patients (3.57\%) in the LA group achieved zero ischemia, while 6 of the 26 patients $(23.08 \%)$ in the LAB group achieved zero ischemia (Table 2 ).

Table 2

Results for the laparoscopic renal tumour enucleation

\begin{tabular}{|llll|}
\hline Item & $\begin{array}{l}\text { LAB group } \\
(\boldsymbol{n}=\mathbf{2 6})\end{array}$ & $\begin{array}{l}\text { LA group } \\
(\boldsymbol{n}=\mathbf{2 8})\end{array}$ & $\boldsymbol{p}$ value \\
\hline Operation time (min) & $88.38 \pm 47.39$ & $98.79 \pm 37.19$ & 0.188 \\
\hline Warm ischemia time (min) & $12.92 \pm 8.38$ & $17.89 \pm 6.73$ & 0.010 \\
\hline Zero ischemia (\%) & $6(23.08 \%)$ & $1(3.57 \%)$ & 0.033 \\
\hline Median blood loss (ml) & $188.31 \pm 170.48$ & $199.89 \pm 140.07$ & 0.394 \\
\hline Intraoperative transfusion (\%) & $2(7.69 \%)$ & $1(3.57 \%)$ & 0.509 \\
\hline Complication (\%) & $0(0 \%)$ & $1(3.57 \%)$ & 0.331 \\
\hline Pseudo capsule damage (\%) & $0(0 \%)$ & $1(3.57 \%)$ & 0.331 \\
\hline
\end{tabular}

In order to further detect the recovery of renal function of patients, the values of serum creatinine, eGFR and ipsilateral renal function (\%) were measured and analysed. We can see that, there was no difference in serum creatinine, eGFR and ipsilateral renal function (\%) values of patients in the LA group and the LAB group before surgery (Table 3). While, after a regular follow-up of 12 months, it was found that the patients in the LAB group had a quicker recovery at 3 months after the operation (eGFR: LAB $98.51 \pm$ $20.23 \mathrm{vs}$. LA $89.74 \pm 15.70 \mathrm{ml} / \mathrm{min} / 1.73 \mathrm{~m}^{2}$; ipsilateral renal function LAB $41.67 \pm 10.11 \% \mathrm{vs}$. LA $36.75 \pm$ $10.30 \%$, Table 3$)$. 
Table 3

Perioperative renal function of different groups

\begin{tabular}{|llll|}
\hline Item & $\begin{array}{l}\text { LAB group } \\
(\boldsymbol{n = 2 6 )}\end{array}$ & $\begin{array}{l}\text { LA group } \\
(\boldsymbol{n}=\mathbf{2 8})\end{array}$ & $\boldsymbol{p}$ value \\
\hline Serum creatinine $(\mathrm{mg} / \mathrm{dl})$ & & & \\
\hline Preoperative & $90.85 \pm 21.31$ & $94.08 \pm 12.59$ & 0.266 \\
\hline 3 month & $94.46 \pm 21.90$ & $102.19 \pm 18.18$ & 0.095 \\
\hline 6 month & $93.46 \pm 20.31$ & $99.46 \pm 16.91$ & 0.142 \\
\hline 12 month & $90.92 \pm 17.11$ & $97.81 \pm 15.39$ & 0.078 \\
\hline eGFR (ml/min/1.73m $\left.{ }^{2}\right)$ & & & \\
\hline Preoperative & $103.20 \pm 24.15$ & $96.23 \pm 12.75$ & 0.101 \\
\hline 3 month & $98.51 \pm 20.23$ & $89.74 \pm 15.70$ & 0.045 \\
\hline 6 month & $98.74 \pm 17.52$ & $91.84 \pm 14.67$ & 0.070 \\
\hline 12 month & $100.94 \pm 17.33$ & $93.18 \pm 14.64$ & 0.048 \\
\hline Ipsilateral renal function (\%) & & & \\
\hline Preoperative & $49.52 \pm 2.50$ & $48.64 \pm 1.73$ & 0.102 \\
\hline 3 month & $41.67 \pm 10.11$ & $36.75 \pm 10.30$ & 0.049 \\
\hline 6 month & $45.88 \pm 7.30$ & $43.03 \pm 6.92$ & 0.084 \\
\hline 12 month & $47.92 \pm 5.47$ & $46.40 \pm 4.67$ & 0.176 \\
\hline & & & \\
\hline
\end{tabular}

\section{Discussion}

Surgical exposure in a narrow operative space is a constant problem in laparoscopic operations. Surgeons have developed many new surgical methods and instruments to improve it, such as the magnetic anchoring, guidance system (MAGS) [5], suture suspension [6], an intra-abdominal exposure instrument [7], and the natural orifice approach [8]. Each method has its pros and cons.

MAGS is a promising complex technique. Levita Magnetics' Surgical System got approval for laparoscopic gallbladder removal from the Food and Drug Administration of the United States in 2016. It could perform the intraperitoneal operation through a single incision (around $30 \mathrm{~mm}$ ). However, to put in the magnetic anchored laparoscope, the surgeon needs a 20-35 mm trocar, which harms the cosmetic effect of the laparoscopy and increases the incision-related incident ratio. In addition, it needs special magnetic anchored laparoscopic instruments, which greatly limits its clinical application [9]. Suture suspension or fixation is a convenient method for laparoscopy surgeons. It needs no additional special 
instruments but traditional laparoscopic instruments, especially a vasocan hollow pneumoperitoneum needle. This technique allows laparoscopic surgeons to suspend any point without adding a trocar or any obvious scar and thus decreases the difficulty of laparoscopic ureteropelvic anastomotic suture [10]. Although it helps to expose the operative space almost without damage to the cosmetic effect of laparoscopy, it does not help clear the liquids in the operative field. The intra-abdominal exposure instrument was a new invention designed by Mr. Qingyi Zhu (CN201620327288.8) [7]. This hairpinshaped intra-abdominal exposure instrument could help expose the operative field by pushing the tissue aside. It is useful in many operations, especially for very narrow and hard-to-expose places, such as single-incision, retroperitoneal, and laparoscopic adrenalectomy. However, it cannot aspirate the liquids in the operative field and, once fixed, is hard and time-consuming to adjust. To operate or retract a specimen through the natural orifice approach is also quite efficient. It uses the natural orifice, such as the urethra [8] or vagina [11], to help the surgeons perform the operation or to retract a specimen. However, it is limited to some special operations, such as radical prostatectomy, or for female patients only.

For some laparoscopic operations, such as partial nephrectomy or simple renal tumor enucleation, the operative space is rather limited and full of liquids, such as blood, urine, and lymph. In addition, operation time, especially the warm ischemia time, is very limited [12]. In such situations, anatomic exposure and liquid aspiration are of equal importance [13]. However, to aspirate liquid and expose the anatomic plane simultaneously, such as the plane between the pseudocapsule and the renal parenchyma, challenges hand skill. The surgeon has two options: (A) To hold the laparoscopic scissors or ultrasound knife in one hand, with the aspirator or forceps in the other hand in an alternative mode. The time to change and position instruments may lengthen the WIT. (B) The surgeon could hold the laparoscopic forceps in one hand for exposure and have an assistant surgeon manipulate the aspirator. In this kind of layout, the forceps, scissors, and aspirator may congest the anatomic plane, making it difficult to operate. The assistant also needs a learning curve to coordinate with the operative surgeon skilfully, which may affect the WIT [14]. In addition, the fourth trocar may add to abdominal wall trauma.

To solve this problem, the $L A B$ was invented. It combines the functions of forceps and aspirator. It has four virtues. (A) It can aspirate liquid and expose the field with a single instrument, thus saving WIT and increasing the surgeon's confidence for a zero ischemia operation. (B) It saves an additional assistant trocar and operative space. (C) Its design permits the surgeon to aspirate and expose the field by himself or herself without additional assistant surgeon, which facilitates the operation. (D) The LAB's material (silicone rubber) helps protect the pseudocapsule of the tumor and lowering the chance of pseudocapsule damage. In simple laparoscopic tumor enucleation, the operator has to substitute forceps for aspirator constantly if just 3 trocars are used. During forceps withdrawal and aspirator insertion, bleeding in the surgical plane continues. Therefore, the surgeon has to repeat the aspirate-expose-cutaspirate procedure constantly, which lengthens the WIT. The LAB has the advantage of exposing and aspirating simultaneously, thus shortening WIT. In our retrospective analysis, the results showed that the LAB shortened WIT, which is the most important factor for postoperative renal function [12]. 
The limitation of our research is its retrospective nature. In the future, prospective randomized research should be conducted. In addition, the LAB could pass a 12-mm trocar only, while the traditional LA needs only a 5 -mm trocar. However, the $12-\mathrm{mm}$ trocar incision could be used for sample retrieval, which would not affect the postoperative cosmetic effect.

\section{Conclusion}

The LAB exposes and aspirates simultaneously by a single instrument without occupying operative space and thus requires only a single surgeon. It is especially suitable for difficult operations that need to be performed in very narrow fields full of liquids such as blood, urine, and lymph. It has the potential to be used in robot-assisted surgery. Our results proved that the LAB can shorten WIT in renal tumor enucleation operations. In addition, it can be applied in many other operations, such as laparoscopic or robot-assisted laparoscopic radical prostatectomy and renal tumor embolus extraction.

\section{Abbreviations}

NSS

nephron-sparing surgery

LAB

laparoscopic aspirator bracket

LA

laparoscopic aspirator

SE

simple enucleation

WIT

warm ischemia time

CT

computed tomography

SD

standard deviation

MAGS

magnetic anchoring, guidance system

\section{Declarations}

\section{Acknowledgements}

Not applicable.

\section{Authors' contributions}


Fengqi Yan, Xiaoliang Dou, Guangfeng Zhu and He Wang contributed to the conception and design of the study and developed the study protocol. Yong Wang, Xiaoliang Dou and Guangfeng Zhu are responsible for the recruitment of subjects. Qisheng Tang, Bo Zhang, Jianjun Ma and Bo Zhao are responsible for the management of the trial, collection and analysis of the data. Yong Wang contributed to the administrative, technical, material support and obtaining funding. All authors contributed to modification of the original protocol and all authors read and approved the final manuscript.

Funding: This study was funded by the New Technology and New Business funding of Tang Du hospital $(2015,2016)$ and supported by grants from the National Natural Science Foundation of China (No.82002686). Mr. Lidong Mi contributed to the artwork of this research.

\section{Availability of data and materials}

Not applicable.

\section{Declarations}

\section{Ethics approval and consent to participate}

Not applicable.

\section{Consent for publication}

Not applicable.

\section{Competing interests}

The authors declare that they have no competing interests.

\section{Author details}

${ }^{1}$ Department of Urology, Tang Du Hospital, Air Force Military Medical University, Xi'an, Shaan'xi Province, P.R. China. 710038

2 Department of Urology, Bao Ji People’s Hospital, Baoji, Shaan'xi Province, P.R. China. 721000

${ }^{3}$ Department of Urology, Xi Jing Hospital, Air Force Military Medical University, Xi'an, Shaan'xi Province, P.R. China. 710032

\section{References}

1. Rassweiler J, Goezen AS, Klein JT, Rassweiler-Seyfried MC. [The future of laparoscopy and robotics in urology]. Aktuelle Urol. 2018;49:488-99. 
2. Cao DH, Liu LR, Fang Y, Tang P, Li T, Bai Y, Wang J, Wei Q. (2017) Simple tumor enucleation may not decrease oncologic outcomes for T1 renal cell carcinoma: A systematic review and meta-analysis. Urol Oncol 35:661 e615-661 e621.

3. Zhao PT, Richstone L, Kavoussi LR. Laparoscopic partial nephrectomy. Int J Surg. 2016;36:548-53.

4. Veccia A, Antonelli A, Uzzo RG, Novara G, Kutikov A, Ficarra V, Simeone C, Mirone V, Hampton LJ, Derweesh I, Porpiglia F, Autorino R. Predictive Value of Nephrometry Scores in Nephron-sparing Surgery: A Systematic Review and Meta-analysis. Eur Urol Focus. 2020;6:490-504.

5. Best SL, Cadeddu JA. Development of magnetic anchoring and guidance systems for minimally invasive surgery. Indian J Urol. 2010;26:418-22.

6. Zhong W, Du Y, Zhang L, Li X, Zhang C, Fang D, Xiong G, He Z, Zhou L. The Application of Internal Suspension Technique in Retroperitoneal Laparoscopic Partial Nephrectomy for Renal Ventral Tumors. Biomed Res Int. 2017;2017:1849649.

7. Lewandowski PM, Leslie S, Gill I, Desai MM. Laparo-endoscopic single-site donor nephrectomy: techniques and outcomes. Arch Esp Urol. 2012;65:318-28.

8. Zhu C, Su J, Yuan L, Zhang Y, Lu ZJ, Su Y, Wang NH, Gu XJ, Zhu QY. Transurethral assistant transumbilical laparoendoscopic single-site radical prostatectomy. Asian J Androl. 2017;19:473-6.

9. Rivas H, Robles I, Riquelme F, Vivanco M, Jimenez J, Marinkovic B, Uribe M. Magnetic Surgery: Results From First Prospective Clinical Trial in 50 Patients. Ann Surg. 2018;267:88-93.

10. Cui X, He YB, Huang WH, Chen L, Chen JC, Zhou CM. (2020) Mini-laparoscopic pyeloplasty to treat UPJO in infants. Minim Invasive Ther Allied Technol:1-6.

11. Park JS, Kang H, Park SY, Kim HJ, Lee IT, Choi GS. Long-term outcomes after Natural Orifice Specimen Extraction versus conventional laparoscopy-assisted surgery for rectal cancer: a matched case-control study. Ann Surg Treat Res. 2018;94:26-35.

12. Pouliot F, Pantuck A, Imbeault A, Shuch B, Calimlim B, Audet JF, Finley DS, Dujardin T. Multivariate analysis of the factors involved in loss of renal differential function after laparoscopic partial nephrectomy: a role for warm ischemia time. Can Urol Assoc J. 2011;5:89-95.

13. Liapis D, de la Taille A, Ploussard G, Robert G, Bastien L, Hoznek A, Vordos D, Abbou C, Salomon L. Analysis of complications from 600 retroperitoneoscopic procedures of the upper urinary tract during the last 10 years. World J Urol. 2008;26:523-30.

14. Dube $H$, Bahler $C D$, Sundaram $C P$. The learning curve and factors affecting warm ischemia time during robot-assisted partial nephrectomy. Indian J Urol. 2015;31:223-8.

\section{Figures}



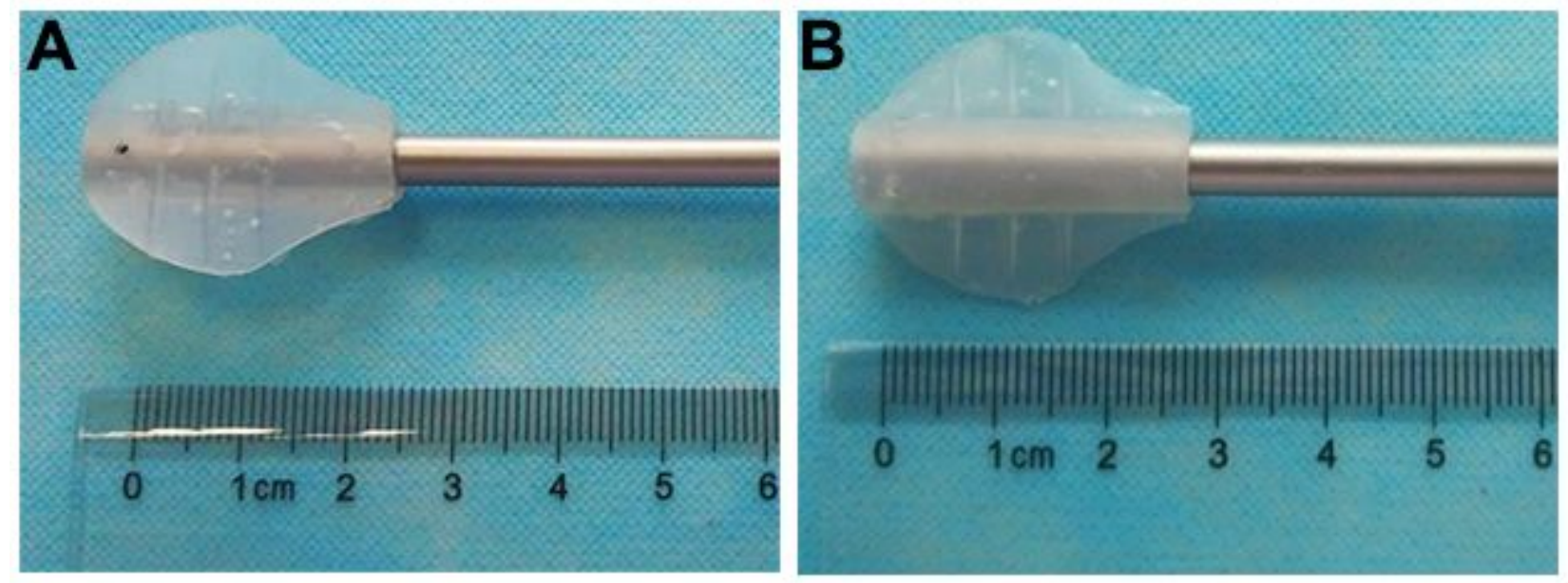

Figure 1

The laparoscopic aspirator bracket (LAB). (A): The front side of the LAB; $(B)$ : The back side of the LAB.

A
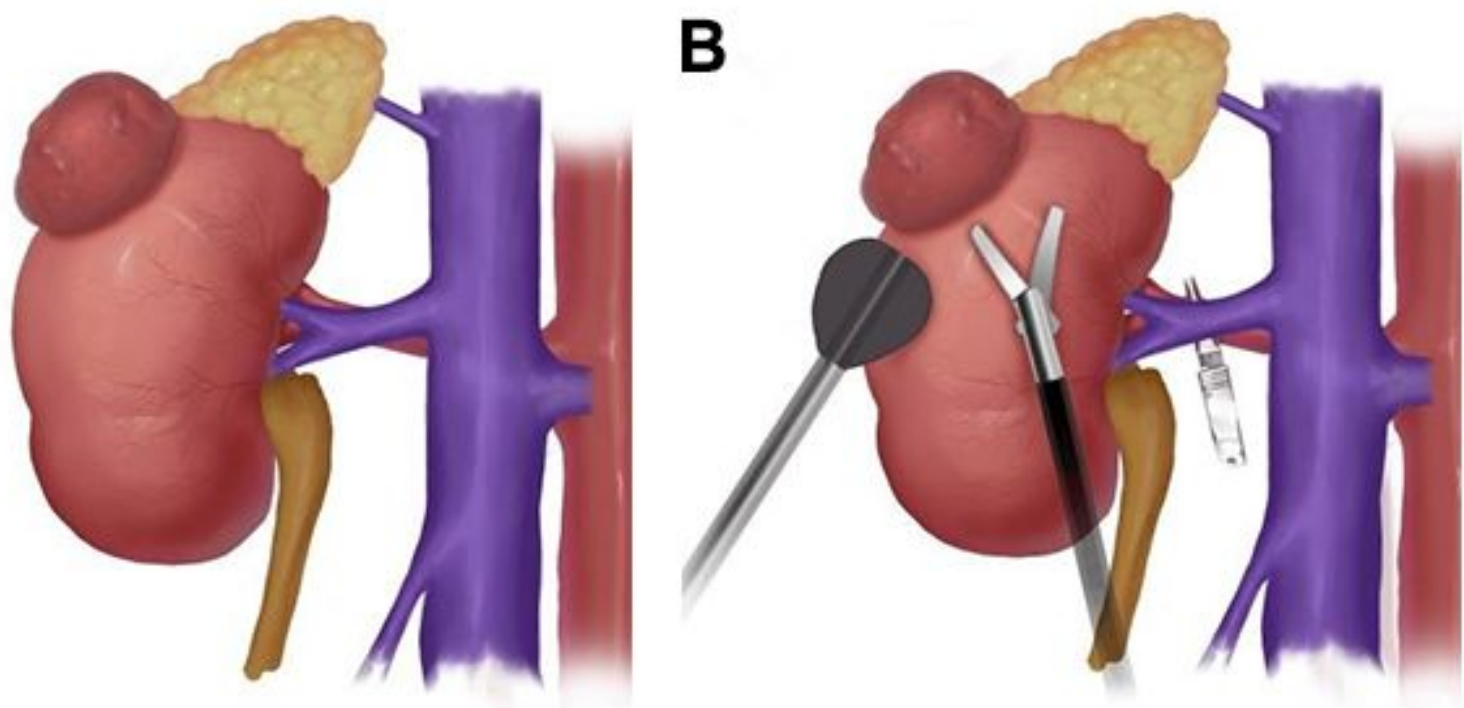

C
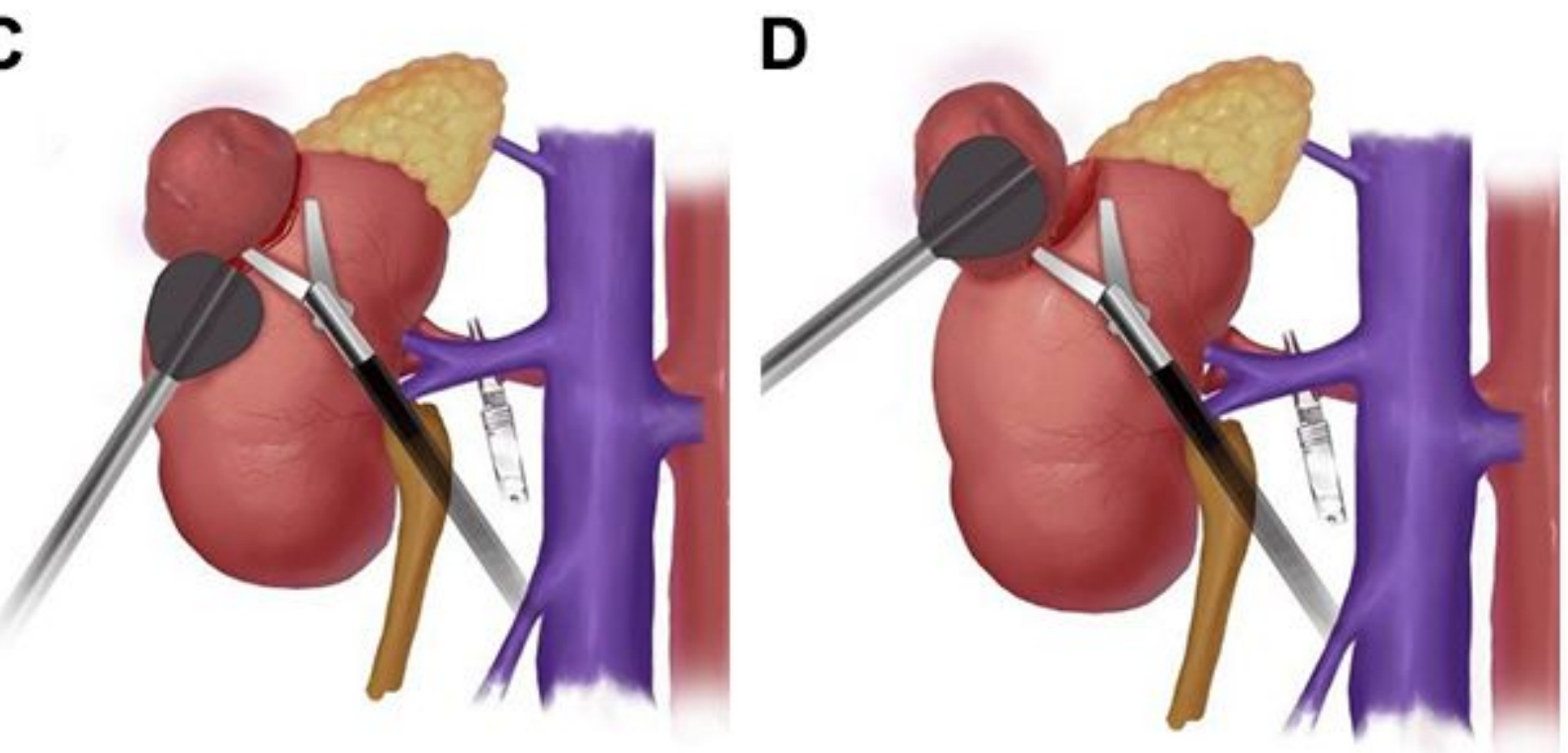


\section{Figure 2}

The schematic diagram of surgical procedure. (A): Schematic diagram of kidney tumor; (B): Schematic diagram of the position of LAB and laparoscopic scissors; (C) Schematic diagram of LAB-assisted scissors to cut open renal capsule; (D) Schematic diagram of LAB combined with scissors cutting and blunt separation of kidney tumors.

\section{Supplementary Files}

This is a list of supplementary files associated with this preprint. Click to download.

- SupplementaryMaterial.mp4 